\title{
Multi-frequency VLBI observations of blazar $1156+295$
}

\section{Wei Zhao*, Xiaoyu Hong, Tao An}

Shanghai Astronomical Observatory, Chinese Academy of Sciences, 200030 Shanghai, China

E-mail: weizhao@shao.ac.cn, xhong@shao.ac.cn, antao@shao.ac.cn

\section{Giuseppe Cimò, Jun Yang}

Joint institute for VLBI in Europe, Postbus 2, 7990 AA Dwingeloo, The Netherlands

E-mail: cimo@jive.nl, junyang@jive.n1

We report the results from multi-epoch observations of 1156+295 with the VLBA at 15, 43 and $86 \mathrm{GHz}$. The source shows a core-jet structure, with a jet extends to the north-east along a curved path at 15 and $43 \mathrm{GHz}$, while the $86 \mathrm{GHz}$ image shows that the inner 0.5 mas jet extends to the southwest. The pc-scale jet of the source can be well fitted by five components (named as C2 to C6 from the outermost inwards) at $15 \mathrm{GHz}$. Two new components C5 and C6 are first detected by our $15 \mathrm{GHz}$ images. Together with our previous observations at $15 \mathrm{GHz}$, the proper motions of the jet components were measured. The total flux density increases by a factor of 2 and the polarization flux density increases by a factor of 1.3 at $15 \mathrm{GHz}$ from epochs 2004.25 to 2005.20. The variations in both total and polarized emission in the core suggest a newly ejected jet component. The $\sim 130^{\circ}$ position angle difference between the $86 \mathrm{GHz}$ jet and $43 \mathrm{GHz}$ jet is likely a result of projection effect. When the jet axis is very close to the line of sight, small change in jet viewing angle could result in large apparent jet bending.

The 9th European VLBI Network Symposium on The role of VLBI in the Golden Age for Radio Astronomy and EVN Users Meeting

September 23-26, 2008

Bologna, Italy

\footnotetext{
${ }^{*}$ Speaker.
} 


\section{Introduction}

The quasar $1156+295(\mathrm{z}=0.729)$ is a good candidate for investigating the AGN nuclear activity and the jet structure change. It has been classified as a Highly Polarised Quasar (HPQ) and an Optically Violent Variable (OVV) source in optical wave length $[1,2,3]$. Three strong $\gamma$-ray flares $\left(\sim 3.9 \times 10^{10} \mathrm{Jy}\right)$ were detected by the EGRET ( Energetic Gamma Ray Experiment Telescope) at energies $>100 \mathrm{MeV}$ from 1992 to 1996, while quiescent $\gamma$-ray emission was undetected [4, 5, 6]. In radio wave length, according to the light curves of $1156+295$ at 4.8, 8.0, and $15 \mathrm{GHz}$ measured at the University of Michigan Radio Astronomy Observatory (UMRAO), several large radio flares were detected from 1980 to 2002. It is interesting that the strongest radio flares show double-peaked feature with time separation of about one year [7].

Previous VLBI observation of 1156+295 exhibited a oscillating jet which can be interpreted as a helical jet moving on a surface of a cone [7]. It shows a north-south direction structure in the VLA $1.4 \mathrm{GHz}$ image [8]. And a single-sided jet extended to the north in PA $=-18^{\circ} \sim-19^{\circ}$ and then bent away to the east $\left(\sim 90^{\circ}\right)$ when it passes through a hot spot about 2 " away from the core, while the diffuse radio emission is also dectected about 2" south of the core in the MERLIN image at $1.6 \mathrm{GHz}$ and VLA image at $5 \mathrm{GHz}$ and $8.5 \mathrm{GHz}$ [7, 9]. The similar jet morphology is also revealed by the MERLIN at $5 \mathrm{GHz}$ and VLA at $22.5 \mathrm{GHz}$, but no counter-jet emission is detected, and only the three main discrete components were detected at $22.5 \mathrm{GHz}$ with VLA [7]. High resolution VLBI images of the source show a "core-jet" structure with an unilateral oscillatory jet on mas scales $[7,9,10,11]$. The jet initially points almost exactly to the north but then bends to the north-east at 3-4 mas from the core, and finally turns about $90^{\circ}$ to the north-west at several tens of milli arcseconds from the core aligning with the direction of the arcsecond-scale jet. A wide range of superluminal velocities of the VLBI jet components from ??c up to 40c have been measured $[7,9,10,12,13]$.

In this paper, we observed the blazar $1156+295$ with the VLBA at three frequencies and four epochs. The observations aims to further investigate the morphology, the polarization and the kinematics of the inner pc-scale jet. We adopt the cosmological model with $H_{0}=73 \mathrm{~km} \mathrm{~s}^{-1} \mathrm{Mpc}^{-1}$, $\Omega_{m}=0.27, \Omega_{\Lambda}=0.73$ throughout the paper.

\section{Observations}

We observed 1156+295 with the VLBA at 15, 43, and $86 \mathrm{GHz}$ at four epochs of 2003 May 10 (2003.36), 2003 July 24 (2003.56), 2004 April 1 (2004.25), 2005 March 13 (2005.20). In each observation, we switched among the three frequencies (from 86 to 43 and $15 \mathrm{GHz}$ ) to optimize the u-v coverage, at $86 \mathrm{GHz}$ a compact quasar was observed before $1156+295$ on every scan to correct the antenna pointing. The total observed time on 1156+295 is about 6 hours on each epoch and allotted on 3 frequencies averagely. The observations were made in dual circular polarization mode, and the total bandwidth is $64 \mathrm{MHz}$. Several scans of J1229+0203 (3C 273), J1310+3220 and OJ 287 which were used for R-L delay offsets correction, instrumental polarization calibration and absolute EVPA calibration respectively were interleaved in the observations of $1156+295$. The raw data were recorded in 2 bits VLBA format and correlated in Socorro, New Mexico. 


\section{Data Reduction}

The data were reduced following the standard procedure of VLBA data reduction in the NRAO software package AIPS (Astronomical Image Processing System). The visibility amplitude of the VLBA data was calibrated using the system temperatures and gain curves monitored during the observations. The atmospheric opacity correction was applied. And then the parallactic angles were determined and removed from the data. Fringe fitting was run on a short scan of $3 \mathrm{C} 273$ to calibrate the residual delays and phase rates. The solutions were then interpolated to calibrate data of all other sources. J 1229+0203 (3C 273) or OJ 287 were used to calibrate the complex bandpass response.

After the routine calibration, a series of polarization-specific calibrations were run on 15 and $43 \mathrm{GHz}$ data: first, we determined the R-L delay difference by running the VLBA procedure VLBACPOL on the source 3C 273 which is intensively polarized. And then, we calibrated the instrumental polarization parameters of the antenna feeds with source $\mathrm{J} 1310+3220$ and then applied the solution to the whole set of data. Finally, the calibration of the absolute polarization angle was made from observations of OJ 287 or J1310+3220. At $15 \mathrm{GHz}$, we used OJ 287 which is comparably compact and stable in this band as the EVPA calibrator. To obtain the correction, we compared it's apparent EVPA with the simultaneous or proximately simultaneous value archived from the MOJAVE/2cm Survey database. At $43 \mathrm{GHz}$, OJ 287 and J1310+3220 were both used. And the corrections were derived by comparing the apparent EVPA with the value of VLA/VLBA Polarization Calibration Resources proximately simultaneous.

After initial calibration, the data were exported into DIFMAP for self-calibration and hybrid mapping.

\section{Results}

\subsection{Pc- and subpc-scale jet structure}

The images of $1156+295$ on four epochs at the three frequencies were obtained and showed a core-jet morphology. The total flux density is dominated by the compact core. Figure 1 shows the images at the three frequencies on the epoch of 2003 July 24 (2003.56).

In $15 \mathrm{GHz}$ images, it shows an oscillating jet to the north (Fig. 1a). The jet tends to diffuse at 5 mas from the core where it bends to the northeast (see Fig. 1a) [7].

On $43 \mathrm{GHz}$ images, it gave us a higher resolution to show the more detail information on the scale less than inner 1 mas (Fig. 1b). The curved jet first goes towards north and then bent to northeast at 0.5 mas north to the core.

On $86 \mathrm{GHz}$ images, there is a core-jet structure extends to south-west. If we believe the strongest component is core, there is a huge misalignment of the jet between $86 \mathrm{GHz}$ and lower frequencies. The misalignment of the jet direction is at least 100 degree. It is probably due to the projection effect. Since the position of the core component is still uncertain, the possibility that the brightest component on the image may be just a bright knot in the jet can not be ruled out. More information are needed for the further study. 


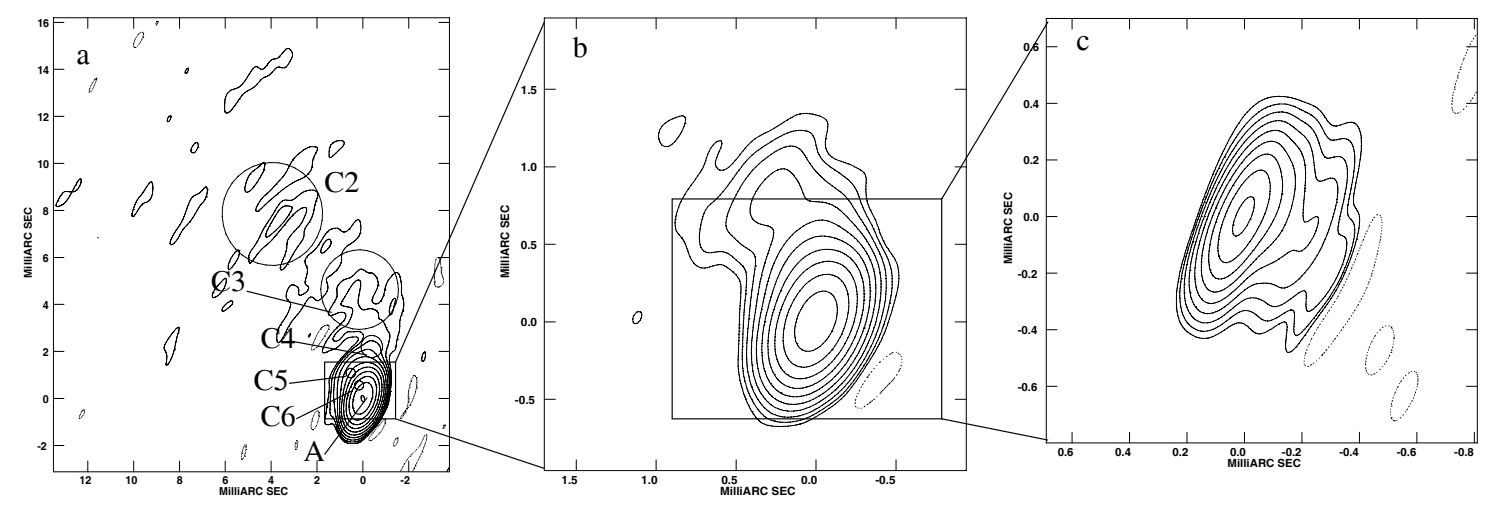

Figure 1: : The VLBA images at the epoch 2003.56. a) $15 \mathrm{GHz}$ image overlapped with six gaussian components ( $\mathrm{C} 2$ to $\mathrm{C} 6$ ). The restoring beam is $1.28 \times 0.69$ mas. The Peak brightness is $1.8 \mathrm{Jy} / \mathrm{Beam}$. The contours are $1.6 \mathrm{mJy} /$ beam $\times(-1,1,2,4, \ldots, 1024)$; b) $43 \mathrm{GHz}$ image. The restoring beam is $0.47 \times 0.27 \mathrm{mas}$. The Peak brightness is $2.2 \mathrm{Jy} /$ Beam. The contours are $2.6 \mathrm{mJy} / \mathrm{beam} \times(-1,1,2,4, \ldots, 512)$; c) $86 \mathrm{GHz}$ image. The restoring beam is $0.28 \times 0.08$ mas. The Peak brightness is $2.6 \mathrm{Jy} / \mathrm{Beam}$. The contours are $8.7 \mathrm{mJy} / \mathrm{beam}$ $\times(-1,1,2,4, \ldots, 256)$

Table 1: Emission Properties of 1156+295

\begin{tabular}{|c|c|c|c|c|c|c|c|c|}
\hline Epoch & Frequency & $\mathrm{S}_{I}^{a}(\mathrm{Jy})$ & $\mathrm{S}_{P}^{b}(\mathrm{mJy})$ & $\mathrm{m}^{c}(\%)$ & Frequency & $\mathrm{S}_{I}^{a}(\mathrm{Jy})$ & $\mathrm{S}_{P}^{b}(\mathrm{mJy})$ & $\mathrm{m}^{c}(\%)$ \\
\hline 2003.36 & $15 \mathrm{GHz}$ & 1.93 & 36.8 & 1.9 & $43 \mathrm{GHz}$ & 1.79 & 38.7 & 2.2 \\
2003.56 & $15 \mathrm{GHz}$ & 2.18 & 42.8 & 2.0 & $43 \mathrm{GHz}$ & 2.42 & 43.8 & 1.8 \\
2004.25 & $15 \mathrm{GHz}$ & 0.73 & 19.1 & 2.6 & $43 \mathrm{GHz}$ & 0.60 & 8.1 & 1.4 \\
2005.20 & $15 \mathrm{GHz}$ & 1.47 & 25.5 & 1.7 & $43 \mathrm{GHz}$ & 1.72 & - & - \\
\hline
\end{tabular}

${ }^{a}$ : the integral flux density; ${ }^{b}$ : the polarized flux density; ${ }^{c}$ : fractional polarization.

\subsection{New apparent superluminal components}

In order to qualitatively analyze the kinematics of the pc-scale jet, we fitted the source emission structure at $15 \mathrm{GHz}$ with six gaussian components [14]. The brightest component (named as 'A'), assuming to be the core, is fitted with an elliptical gaussian. The jet components (named as ' $\mathrm{C} 2$ ' to 'C6' from outermost inwards) are fitted with circular gaussian components. The components C2, C3 and C4 also appear in VLBI images in Hong et al. (2004). The components C5 and

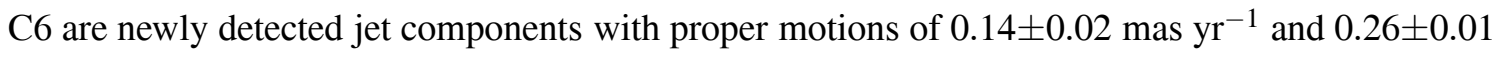
mas $\mathrm{yr}^{-1}$, which correspond to $4.9 \pm 0.7 c, 9.2 \pm 0.4 c$, respectively $\left(\mathrm{q}_{0}=0.5, H_{0}=65 \mathrm{~km} \mathrm{~s}^{-1} \mathrm{Mpc}^{-1}\right)$.

\subsection{Polarization at 15 and $43 \mathrm{GHz}$}

Table 1 lists the total flux density, the polarized flux density, and fractional polarization (of the overall source) on four epochs for 15 and $43 \mathrm{GHz}$. Significant variations on both total (Stokes I) and polarized flux density are found at 15 and $43 \mathrm{GHz}$. The polarization was not detected at $43 \mathrm{GHz}$ at the epoch of 2005.20 due to lower signal-to-noise ratio. 
The total flux density is enhanced from 0.73 to $1.47 \mathrm{Jy}$ at $15 \mathrm{GHz}$ (or a factor of 2.0) and from 0.60 to $1.72 \mathrm{Jy}$ at $43 \mathrm{GHz}$ (a factor of 2.9), on the other hand the polarized flux density also increases from 19.1 to $25.5 \mathrm{mJy}$ (or a factor of 1.3) at $15 \mathrm{GHz}$. The increase of total I and polarized flux density in the core may be related to the creation and ejection of a new jet component [13], although the new jet component is still blended with the core on the observing epoch. Change of EVPA is missed in this paper.

It is clear that the total flux densities and the polarized flux density increased from the epoch of 2003.36 to the epoch of 2003.56, then they deceased to the epoch of 2004.25 at its lowest status of the four epoch. After that the source seems to outburst again.

The total flux density varied by a factor of $3(0.73$ to $2.18 \mathrm{Jy})$ and the polarized flux density varied by a factor of 1.3 ( 19.1 to to $25.5 \mathrm{mJy}$ ) at $15 \mathrm{GHz}$, and they both changed by a factor of 4 at $43 \mathrm{GHz}$.

The variation in the core area may related to the outburst and ejected of a new jet component.

\section{Summary}

The VLBA images of $1156+295$ at 15,43 , and $86 \mathrm{GHz}$ show a core jet structure with an oscillating jet to the northeast on scale large than 1 mas, while it extends to the southwest on scale of $\mathbf{0 . 2}$ mas. Two new jet components were detected with superluminal velocities of $4.9 \pm 0.7 c$, $9.2 \pm 0.4 c$. The variations of both total and polarized flux density could be related to the birth of a new jet component.

\section{Acknowledgements}

This research was supported by the Natural Science Foundation of China (NSFC10473018, 10503008). The National Radio Astronomy Observatory is a facility of the National Science Foundation operated under cooperative agreement by associated Universities, Inc.

\section{References}

[1] Wills, B. J., Wills, D., Breger, M., Antonucci, R. R.,\& Barvainis, R. E.1983, ApJ, 274, 62

[2] Wills, B. J., Pollock, J. T., Aller, H. D., et al. 1992, ApJ, 398, 454

[3] Glassgold, A. E., Bergman, J.M., Huggins, P. J., et al. 1983, ApJ, 274, 101

[4] Thomposon, D. J., Bertsch, D. L., Dingus, B. L., et al. 1995, ApJS, 101, 259

[5] Mukherjee, R., Bertsch, D. L., Bloom, S. D., et al. 1997, ApJ, 490, 116

[6] Hartman, R. C., Bertsch, D. L., Bloom, S. D., et al. 1999, ApJS, 123, 79

[7] Hong, X. Y., Jiang, D.R., Gurvits, L.I., et al. 2004, A\&A, 417, 887

[8] Antonucci, R. R., \& Ulvestad, J. S. 1985, ApJ, 294, 158

[9] McHardy, I. M. et al., 1990, MNRAS, 246.305

[10] Piner, B. G. , \& Kingham, K. 1997, ApJ, 485

[11] Hong, X. Y., Jiang, D. R., Gurvits, L. I., et al. 1999, NewAR, 43, 699 
[12] McHardy, I. M., Marscher, A. P., Gear, W. K., et al. 1993, MNRAS,261, 464

[13] Jorstad, S. G., Marscher, A. P., Mattox, J. R., et al. 2001, ApJs, 134, 181

[14] Zhao, W., Hong, X. Y., An, T., et al. 2009, Proceedings of the 10th Asian-Pacific Regional IAU Meeting, in press 